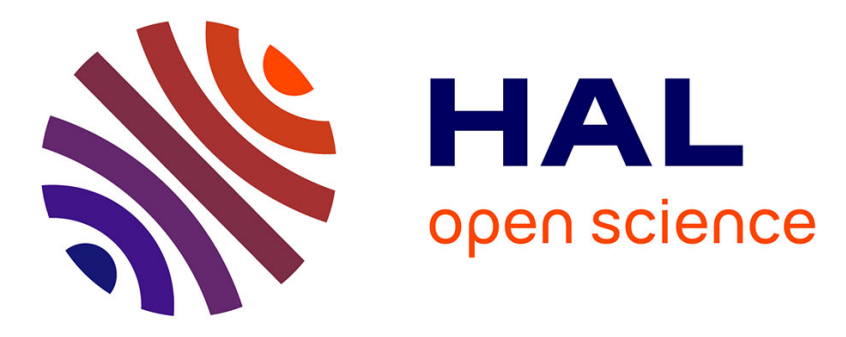

\title{
Les glaciers noirs à l'épreuve du climat (Prix Prud'homme 2019)
}

Fanny Brun, Étienne Berthier, Patrick Wagnon

\section{To cite this version:}

Fanny Brun, Étienne Berthier, Patrick Wagnon. Les glaciers noirs à l'épreuve du climat (Prix Prud'homme 2019). La Météorologie, 2020, 109, pp.016. 10.37053/lameteorologie-2020-0042 . insu03319263

\section{HAL Id: insu-03319263 \\ https://hal-insu.archives-ouvertes.fr/insu-03319263}

Submitted on 12 Aug 2021

HAL is a multi-disciplinary open access archive for the deposit and dissemination of scientific research documents, whether they are published or not. The documents may come from teaching and research institutions in France or abroad, or from public or private research centers.
L'archive ouverte pluridisciplinaire HAL, est destinée au dépôt et à la diffusion de documents scientifiques de niveau recherche, publiés ou non, émanant des établissements d'enseignement et de recherche français ou étrangers, des laboratoires publics ou privés.

\section{(c)(1)}

Distributed under a Creative Commons Attribution| 4.0 International License 


\title{
Prix Prud'homme 2019 Les glaciers noirs à l'épreuve du climat
}

\author{
Fanny Brun', Étienne Berthier ${ }^{2}$, Patrick Wagnon ${ }^{1}$ \\ 1. Institut des géosciences de l'environnement, Université Grenoble Alpes / \\ CNRS / IRD, Saint-Martin-d'Hères
}

2. Laboratoire d'étude en géophysique et océanographie spatiales, Université Paul Sabatier / CNRS, Toulouse

fanny.brun@univ-grenoble-alpes.fr

\section{Résumé}

Les glaciers noirs, dont la langue est couverte de débris rocheux, ont un équilibre avec le climat qui diffère de celui des glaciers blancs, en raison du caractère isolant de leur couverture détritique. L'objectif est ici d'observer les changements récents (sur des périodes de 3 à 16 ans) de certains glaciers noirs pour mieux comprendre les processus qui déterminent leur fonte et pour comparer leur évolution à celle des glaciers blancs.

\section{Abstract}

Influence of debris on the mass balance of high mountain Asia glaciers

Debris covered glaciers respond differently from debris-free glaciers to climate change, due to the insulation properties of debris. Here we observe recent and short (3 to 16 years) changes of selected debris-covered glaciers. We aim to better understand melting processes and compare the evolution of debris-free and debris-covered glacier changes. d'érosion glaciaire et périglaciaire remobilisés par l'eau et le vent. Ils se sont déposés soit dans la zone d'accumulation, soit dans la zone d'ablation du glacier. Si les débris sont déposés dans la zone d'accumulation, ils sont enfouis sous les couches de neige puis de glace, puis transportés avant de réémerger dans la zone d'ablation. S'ils sont déposés dans la zone d'ablation, ils s'accumulent sur les débris déjà présents ou sur la glace et sont transportés vers le front du glacier.

Les débris se retrouvent ainsi quasi exclusivement sur les langues d'ablation des glaciers, lieux où la fonte saisonnière est importante. Les débris viennent alors moduler la fonte de la glace qu'ils recouvrent en fonction de leur épaisseur. Ils ont donc une influence potentielle sur les processus d'ablation des glaciers et, par conséquent, une influence sur la relation glacier-climat très grande au regard de la surface relativement restreinte qu'ils occupent.

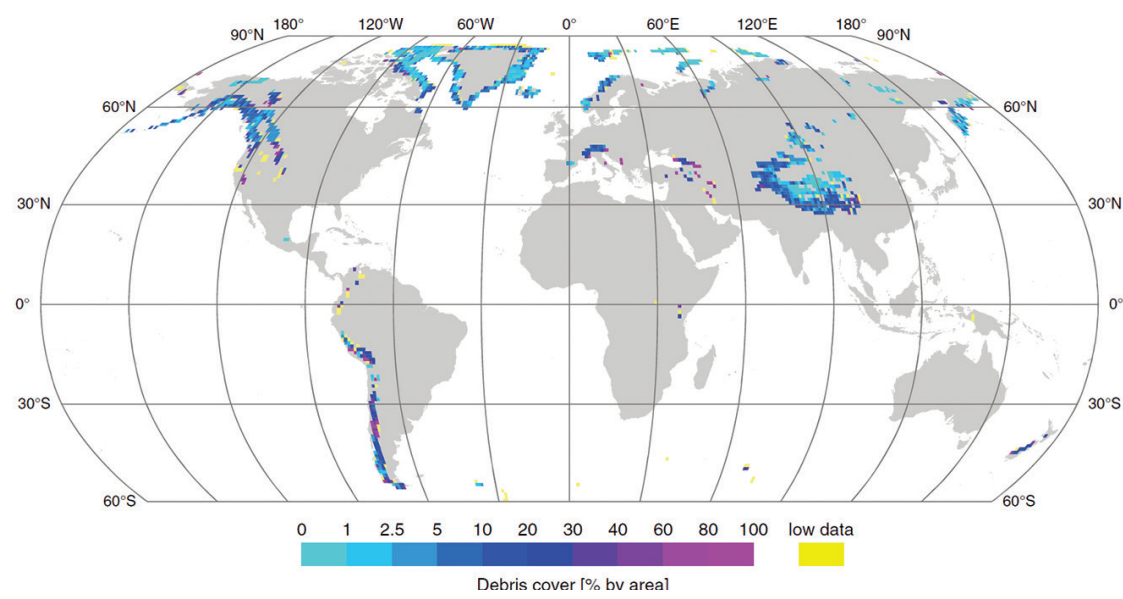

Figure 1. Répartition des glaciers noir dans le monde. Chaque trapèze coloré représente la fraction de couverture détritique sur les glaciers pour une région de $1^{\circ}$ par $1^{\circ}$. Source : Scherler et al. (2018). 

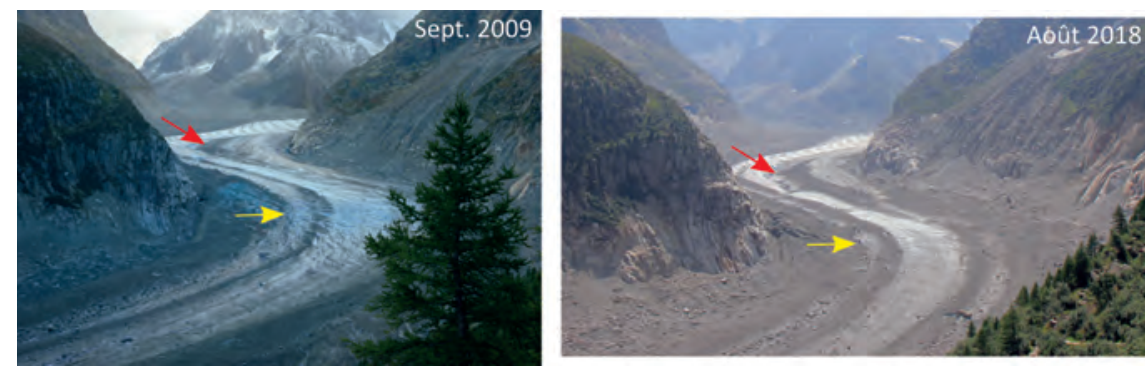

Figure 2. Évolution de la surface de la Mer de Glace (Chamonix) entre septembre 2009 (gauche) et août 2018 (droite). Les flèches pointent sur des localisations où l'augmentation de la couverture de débris est particulièrement marquée. Photos : Bruno Jourdain.

Un dernier argument en faveur de l'étude de ces glaciers dits noirs est leur importance future. Dans le cadre du réchauffement climatique, les glaciers s'amincissent et donc ralentissent. Cela est observé depuis plusieurs années et continuera très probablement dans le futur. Cette baisse de la dynamique glaciaire favorise l'émergence de débris qui ne sont plus évacués par le glacier. En conséquence, la couverture de débris augmente et continuera d'augmenter. Ce phénomène est déjà visible dans le cas emblématique de la Mer de Glace, qui est passée d'une langue blanche à une langue couverte de débris en quelques décennies (figure 2).

Il est donc clair que les glaciers noirs vont gagner en importance dans le futur. D'une part parce que les surfaces couvertes de débris vont s'accroître au moins en valeur relative par rapport à la surface totale des glaciers du monde, d'autre part parce que ces surfaces sont situées à basse al titude, là où la fonte est intense. Comprendre comment cette couche de débris influence la fonte est donc une nécessité. Ce besoin est particulièrement prégnant dans les hautes montagnes d'A sie, où les glaciers ont une grande importance pour la ressource en eau (B erthier et al ., 2016). À I'horizon 2100, un à deux tiers des glaciers de ces hautes montagnes auront disparu, selon les scénarios d'émission de gaz à effet de serre envisagés (K raaijenbrink et al., 2017). Pour les bassins-versants de ces glaciers, cela se traduit dans un premier temps par une augmentation du débit des rivières en raison de l'augmentation de la fonte, irrémédiablement suivie par une diminution lorsque les surfaces englacées deviennent trop faibles. Quand ce pic de débit aura-t-il lieu ? Comment l'eau de fonte sera-t-elle distribuée au cours des saisons ? Voilà des questions cruciales auxquelles il est encore difficile de répondre avec précision. A insi, il ne sera jamais pleinement possible d'estimer l'évolution future des glaciers sans prendre en compte leur couverture détritique, l'évolution future de cette couverture et les processus de fonte qui leur sont propres.

Dans cet article, nous décrivons les changements récents des glaciers noirs des hautes montagnes d'A sie. En particulier, nous avons choisi de travailler à plusieurs échelles, en quantifiant précisément un processus de fonte sur un seul glacier, puis en analysant les changements de masse de toute une population de glaciers.

\section{Les processus d'ablation spécifiques aux glaciers couverts}

Pour comprendre la relation entre glaciers noirs et climat, il faut d'abord chercher à comprendre comment la présence de débris influence la fonte de la glace sous les débris à travers des processus spécifiques aux glaciers noirs.

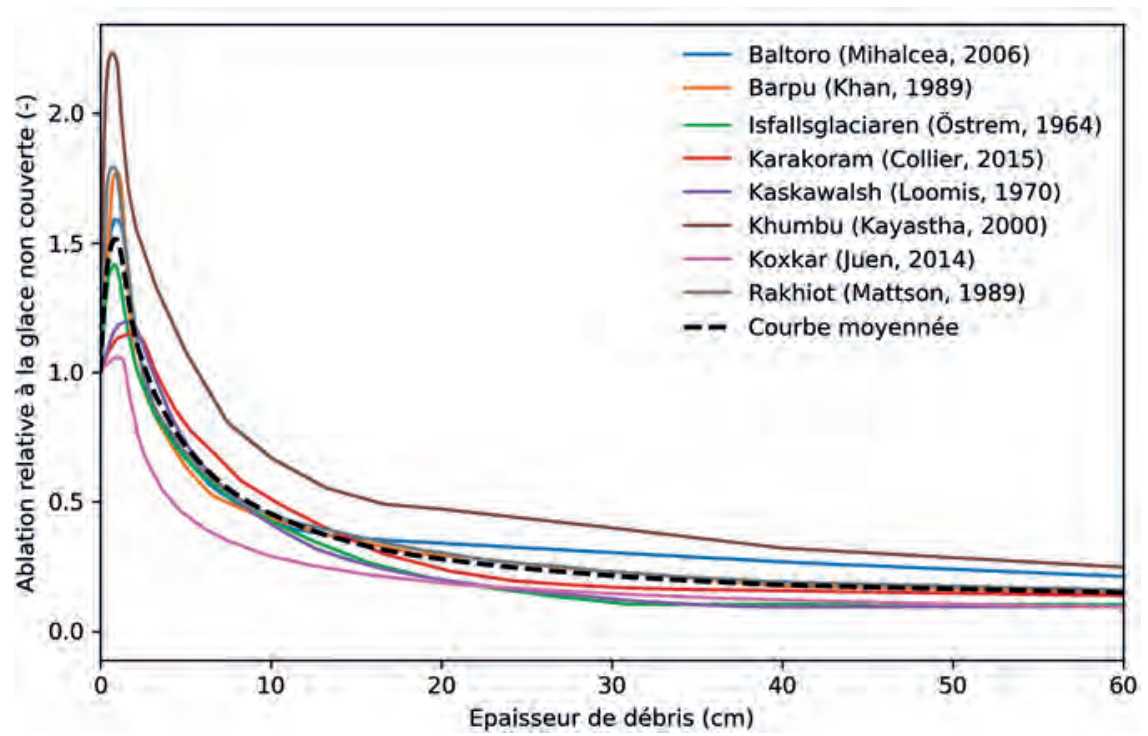

Figure 3. Compilation de courbes d'Østrem montrant la relation entre épaisseur de débris rocheux (en $\mathrm{cm}$ ) et fonte (ici normalisée par rapport à la fonte de la glace non couverte). Les données ont été compilées par Philip Kraaijenbrink (université d'Utrecht).

\section{Fonte sous une couche de débris}

La présence de débris rocheux influence la fonte de la glace sousjacente à travers la modification du bilan d'énergie à la surface de la glace. La mesure directe de la fonte sous les débris est assez difficile à réaliser sur de grandes durées, car elle implique de mesurer de manière répétée l'émergence de jalons insérés dans la glace sous les débris. II faut donc excaver le mélange de sable et de blocs partiellement gelés jusqu'à atteindre la glace en dessous pour y forer un trou, y insérer un jalon et replacer les débris sur ce dernier. Les jalons qui émergent des débris ont tendance à se briser à cause des blocs qui les heurtent. Ces mesures sont le plus souvent conduites pendant la saison d'ablation (été dans l'hémisphère Nord) et sur de courtes durées, typiquement quelques semaines. Les ablations mesurées sous les débris vont de 0 à $4 \mathrm{~cm}$ équivalent-eau par jour.

Une compréhension assez intuitive de l'influence des débris est qu'une fine couche de débris a tendance à accélérer la fonte par effet d'albédo : les débris sont plus sombres que la glace, donc ils absorbent davantage de rayonnement solaire et réchauffent la surface du glacier. À l'inverse, une couche plus épaisse de débris va ralentir la fonte par effet isolant. $L$ a relation entre épaisseur de débris et fonte est connue sous le nom de courbe d'Østrem ( $\varnothing$ strem, 1959 ; figure 3), nommée d'après le célèbre glaciologue norvégien Gunnar Østrem (1922-2020). La compétition entre ces deux effets a été assez 
largement observée et modélisée à petite échelle. Même si de nombreuses incertitudes demeurent et s'il existe une grande variabilité entre les différents sites d'études, on peut retenir que l'effet isolant des débris domine pour des épaisseurs de débris supérieures à 3-4 cm et, que pour des débris de plus de 20-25 cm d'épaisseur, l'ablation est réduite de $75 \%$ environ par rapport à de la glace propre.

Des épaisseurs de débris dépassant plusieurs dizaines de centi mètres sont très fréquemment observées sur les langues des glaciers couverts, notamment pour un certain nombre de glaciers des hautes montagnes d'A sie qui ont été beaucoup étudiés ces dernières années (dans les vallées de l'Everest ou du Langtang au Népal notamment). L'épaisseur des débris est aussi particulièrement laborieuse à mesurer, puisqu'elle consiste à creuser à la pelle jusqu'à la glace. Des équipes de recherche travaillent à spatialiser cette mesure grâce à des méthodes géophysiques, comme l'utilisation de radars, mais ces méthodes ont été déployées sur un nombre très restreint de glaciers et n'ont pas toujours abouti. Les répartitions spatiales des épaisseurs de débris sont donc peu connues et, à l'heure actuelle, il est difficile d'appliquer des modèles pour modéliser la fonte à l'échelle d'une langue glaciaire, même s'il semble y avoir consensus sur le fait que l'effet isolant des débris domine, sauf dans certaines zones, comme à la transition entre glace couverte et non couverte (figure 4).

\section{Augmentation locale de la fonte}

L es langues couvertes de débris ont une surface très chahutée et hétérogène. Çà et là, des talus raides (pente $>30-35^{\circ}$ ) émergent et laissent apparaître la glace, dépourvue de débris, qui glissent au pied. Ces talus sont appelés falaises de glace (figure 5). Depuis la fin des années 1990, des équipes de recherche soupçonnent ces falaises d'être des zones préférentielles d'échange d'énergie, et donc de fonte, entre l'atmosphère et la glace (Sakai et al., 1998). Cela a été observé de façon qualitative sur le terrain, où des retraits spectaculaires de falaises de 5 à $10 \mathrm{~m}$ par an ont été mesurés. Par la suite, des mesures plus précises, réalisées à l'aide de jalons de $2 \mathrm{~m}$ de long insérés dans la glace, perpendiculairement à la surface des falaises, ont permis de mesurer des taux de fonte de 1 à $10 \mathrm{~cm}$ équivalent-eau par jour. Ces mesures montrent que les falaises sont bien des hotspots de fonte.

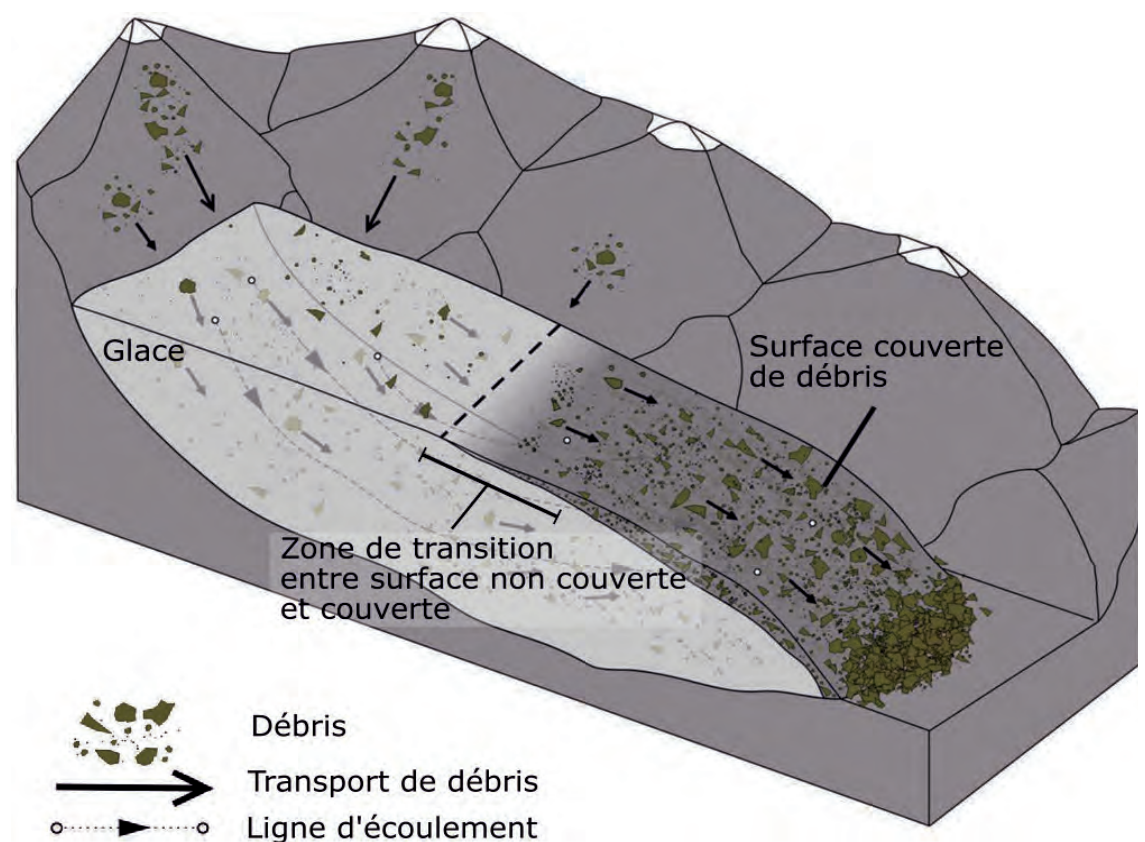

Figure 4. Schéma d'un glacier couvert de débris. D’après Evatt et al. (2015).

Mais comme elles n'occupent que quelques pourcents de la surface réelle des glaciers, il est nécessaire de quantifier leur contribution totale à l'ablation.

La topographie chahutée permet aussi le développement de petits lacs, connectés entre eux par un réseau hydrographique à la surface et à l'intérieur du glacier. Les lacs facilitent le transfert d'énergie de l'atmosphère vers la glace, car ils ont un albédo faible et transportent efficacement l'énergie entre l'atmosphère, l'eau et la glace grâce au mélange turbulent. L'eau liquide circule également sous forme de chenaux et de conduits à l'intérieur du glacier. Cette circulation contribue également à la fonte du glacier.

Dans ce travail, nous nous sommes focalisés sur la fonte des falaises, que nous avons quantifié sur un glacier

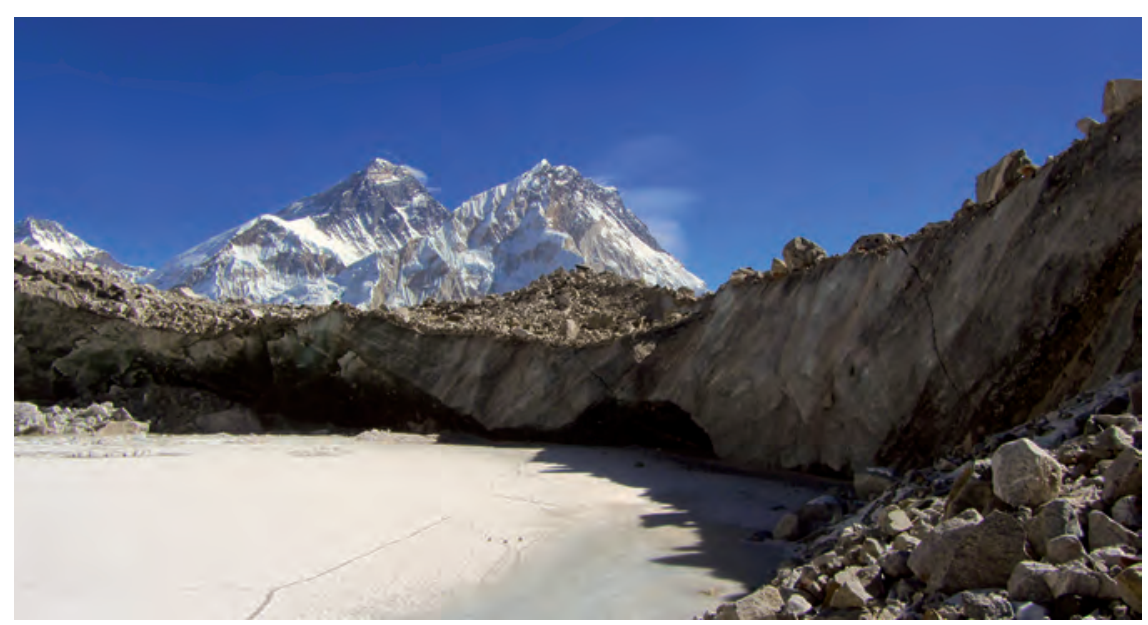

Figure 5. Falaise de glace bordant un lac supraglaciaire sur le glacier du Changri Nup. Les sommets de I'Everest (à gauche) et du Nuptse (à droite) sont visibles en arrière-plan. Photo : Fanny Brun. situé non loin de l'Everest. Cette quantification a nécessité des campagnes de terrain longues (4 à 5 semaines) et répétées chaque année.

\section{Contribution des falaises de glace à la fonte}

\section{Site d'étude : le glacier Changri Nup}

L e glacier retenu pour la quantification des falaises à la fonte est le glacier du Changri Nup, situé dans la région de l'Everest (figure 6). II a une superficie de $2,7 \mathrm{~km}^{2}$, dont $1,5 \mathrm{~km}^{2}$ sont recouverts de débris à une altitude comprise 


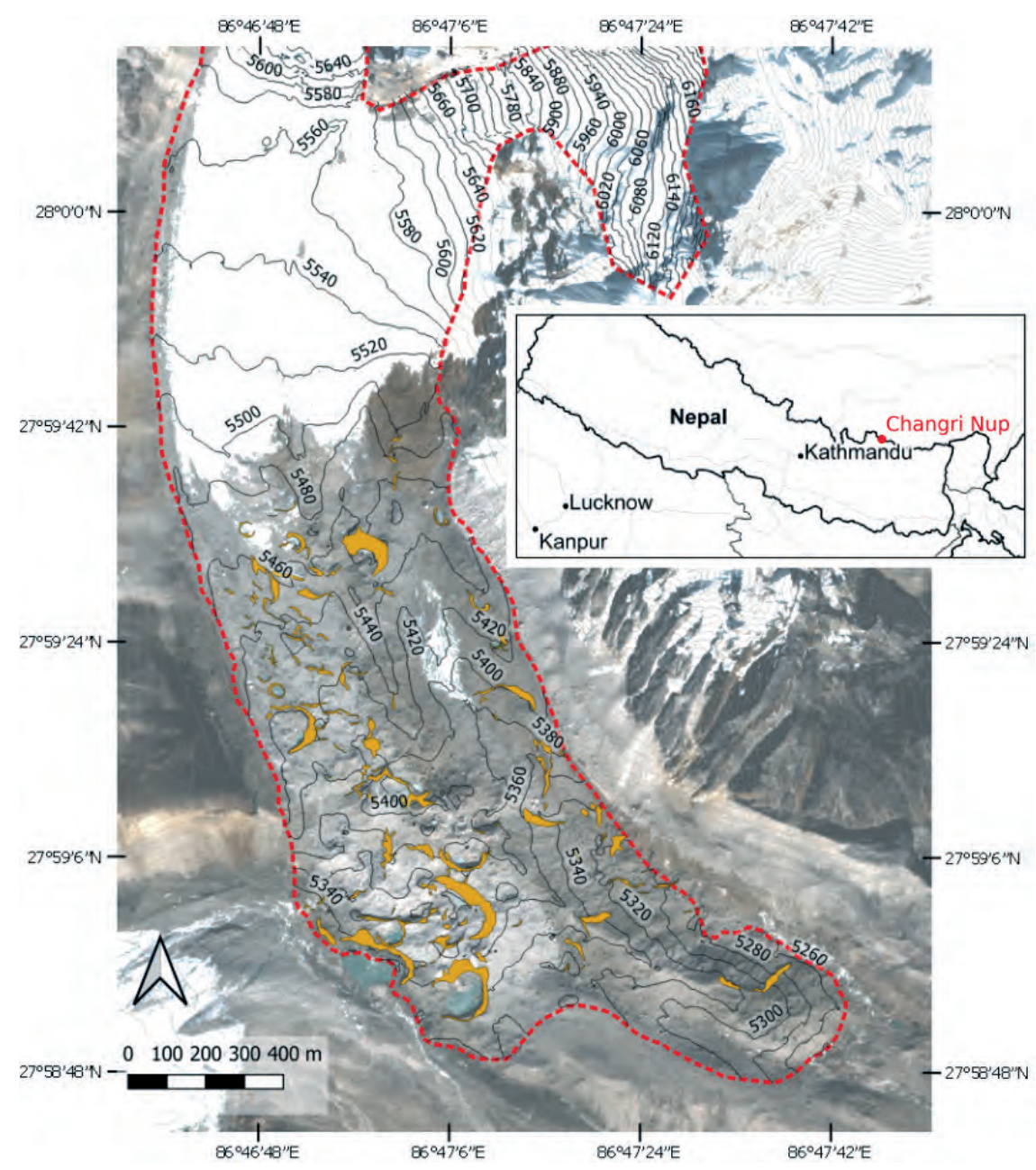

Figure 6. Carte du glacier du Changri Nup (contour rouge). Les formes orange représentent les projections planisphériques des falaises cartographiées à l'automne 2016. Le fond est une orthoimage Pléiades de novembre 2016. C Cnes 2016, Distribution Airbus D\&S

entre 5500 et $5300 \mathrm{~m}$ d'altitude. Les falaises n'étant pas parfaitement verticales, il est possible de mesurer leur surface projetée : elle occupe environ 7 à $8 \%$ de la surface couverte de débris. Le glacier du Changri Nup est suivi depuis 2011. Des jalons mesurés chaque année permettent de connaître le bilan de masse localement à la fois dans des zones couvertes et non couvertes de débris. En 2011, nous avons mesuré l'épaisseur du glacier le long d'un profil transversal à environ 5525 m, juste en amont de la zone de transition entre glace propre et glace couverte de débris.

Traditionnellement, en glaciologie, le bilan de masse mesuré en quelques points précis est ensuite spatialisé par interpolation et extrapolation pour obtenir une variation de masse à l'échelle de tout un glacier. Cette méthode n'est pas applicable dans le cas des glaciers couverts, car la surface est trop hétérogène et la variabilité spatiale du bilan de masse entre les jalons est trop grande. Une méthode alternative est donc de s'appuyer sur
Pour un glacier, la variation d'épaisseur locale $\left(\frac{\partial H}{\partial t}\right)$ dépend à la fois du bilan de masse de surface (accumulation et ablation) et de l'advection de la topographie par l'écoulement du glacier. Elle s'écrit :

$$
\frac{\partial H}{\partial t}=\frac{\dot{b}_{s}}{\rho_{s}}+w_{s}-u_{s} \frac{\partial H}{\partial x}-v_{s} \frac{\partial H}{\partial y}
$$

où $H$ est l'altitude de la surface du glacier, $\frac{\dot{b}_{s}}{\rho_{s}}$ le bilan de masse de surface divisé par la $\rho_{s}$
masse volumique (la glace dans notre cas), $w_{s}$ de l'écoulement) de la glace (voir encadré). Pour corriger la dynamique, nous avons évalué la vitesse verticale de la glace indirectement, grâce à la mesure du flux de glace à travers la section transversale d'épaisseur connue située juste en amont de la zone couverte (V incent et al., 2016).

\section{Réduction de l'ablation par les débris et contribution des falaises}

Deux résultats principaux se dégagent des mesures conduites sur le glacier du Changri Nup. En extrapolant les gradients de bilan de masse de surface d'un glacier blanc voisin aux mesures d'ablation de la partie non couverte de débris du glacier du Changri Nup, il a été possible de montrer que c'est bien l'effet isolant des débris qui domine. Si la langue du Changri Nup n'était pas couverte de débris, elle aurait perdu 2,5 fois plus de masse entre 2011 et 2015 (V incent et al., 2016). En tirant profit de la très haute résolution spatiale des MNT drone $(50 \mathrm{~cm})$ et des M NT Pléiades $(2 \mathrm{~m})$, nous avons aussi pu quantifier la contribution des falaises de glace à cette ablation. A u cours des deux saisons étudiées (20152016 et 2016-2017), elles ont contribué à environ un quart de l'ablation de la langue. Cela montre que, malgré une ablation localement trois à quatre fois plus forte que pour la glace couverte de débris, la faible surface occupée par les falaises ne permet pas de compenser la réduction de la fonte due aux débris pour atteindre une ablation comparable à celle d'un glacier blanc (Brun et al., 2018).

Cette étude montre la belle complémentarité entre les mesures de terrain (mesures d'épaisseur de glace et d'ablation) et les mesures par satellite (mesures de topographie et de vitesse de surface).

\section{Évolution de la topographie du glacier : bilan de masse et écoulement}

la vitesse verticale, et $u_{s}$ et $v_{s}$ les composantes selon $x$ et $y$ de la vitesse horizontale. Les variations temporelles de la surface du glacier dépendent donc à la fois du bilan de masse de surface (et de la densité) et de la dynamique du glacier. Cela est à garder en tête quand on analyse une carte de changement d'altitude du glacier. Par contre, si cette équation est intégrée sur une surface fermée, comme le contour d'un glacier, le terme dynamique devient nul et le changement d'épaisseur moyen est bien égal au bilan de masse divisé par la masse volumique. 


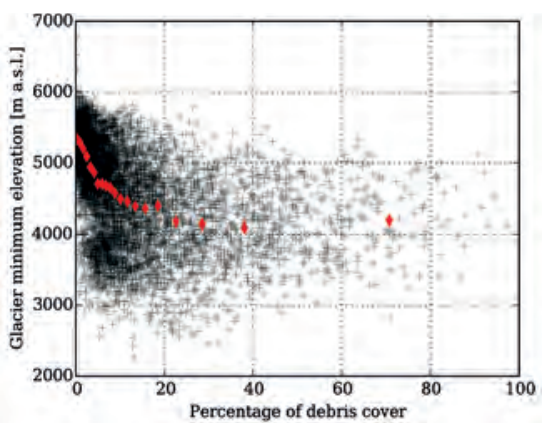

Figure 7. Altitude minimale de plus de 6000 glaciers en fonction du pourcentage de couverture détritique. Les losanges rouges montrent les valeurs médianes pour 20 classes de la distribution de la couverture détritique comprenant chacune $5 \%$ des données.

En revanche, elle n'est réalisable que sur un nombre très limité de glaciers, car il n'existe pratiquement pas de mesures d'épaisseur de glace pour les glaciers couverts. Cela nous empêche donc de généraliser ce que nous avons montré dans le cas du glacier du Changri Nup, à savoir que la fonte sous les débris est plus faible que la fonte de la glace non couverte de débris située à la même altitude. Par contre, le fait que les langues couvertes de débris atteignent des altitudes inférieures à celles des langues non couvertes (figure 7) permet de confirmer indirectement que l'effet isolant des débris domine sur les zones de fonte préférentielles comme les falaises de glace.

II reste à interpréter ces observations dans un contexte climatique, c'està-dire de se demander quelle est la réponse des glaciers noirs à une augmentation de température. Pour cela, nous avons choisi de quantifier l'évolution récente des glaciers des hautes montagnes d'A sie entre 2000 et 2016.

\section{Influence \\ de la couverture détritique sur une population de glaciers}

La suite de ce travail a été de considérer l'ensemble des glaciers des hautes montagnes d'A sie comme une population de glaciers au sein de laquelle on peut voir des différences entre sous-populations (par exemple entre glaciers noirs et glaciers blancs). Nous nous sommes focalisés sur la période 2000-2016, qui correspond à une bonne disponibilité en termes d'images satellitaires. L es différents ingrédients utilisés dans cette étude sont : les bilans de masse individuels des glaciers, l'étendue de la couverture détritique pour chaque glacier et les paramètres morphologiques des glaciers dérivés à partir d'un M NT de référence. La cartographie de l'étendue des débris nous a été fournie par des collègues de l'université d'U trecht (Pays-B as) et les paramètres morphologiques sont dérivés directement à partir du MNT Shuttle Radar Topographic M ission (SRTM ).

\section{Bilans de masse géodésiques à l'échelle} d'une chaîne de montagnes

Les bilans de masse individuels des glaciers des hautes montagnes d'A sie ont été obtenus par traitement automatique de plus de 50000 M NT dérivés d'images stéréoscopiques acquises par le satellite Aster (Advanced Spaceborne Thermal Emission and Reflection Radiometer). Lorsque I'on cherche à calculer le bilan de masse d'un glacier complet, il n'est pas nécessaire de connaître l'épaisseur du glacier, les variations d'épaisseur suffisent, à condition de prendre le glacier dans sa totalité (contrairement au cas de la langue d'ablation du Changri Nup ; voir encadré). À partir de ces données, nous avons produit la première estimation quasi exhaustive $d u$ bilan de masse des glaciers des hautes montagnes d'A sie à $-16,3 \pm 3,5 \mathrm{G}$ t/an, soit un amincissement moyen de 0,18 $\pm 0,04$ m équivalent-eau/an pour la période de 2000-2016 (B run et al., 2017).

II existe une grande variabilité régionale à l'échelle des hautes montagnes
d'A sie (figure 8). Dans certaines régions, les glaciers connaissent des pertes de masse très fortes comme dans le Nyainqentanglha (situé à la frontière entre l'I nde et la $C$ hine, à l'est de l'H imalaya), avec des changements d'épaisseur de - 0,62 $\pm 0,23$ m équivalent-eau/an, alors que les glaciers sont à l'équilibre dans d'autres régions, voire gagnent de la masse, comme dans le $\mathrm{K}$ unlun Shan $(+0,14 \pm 0,08 \mathrm{~m}$ équivalent-eau/an). Ces forts contrastes entre les régions s'expliquent en partie par des sensibilités différentes des glaciers aux changements de températures et/ ou de précipitations, qui dépendent essentiellement de la climatologie (Sakai et Fujita, 2017). Pour s'affranchir de ce signal, nous avons réparti les glaciers des hautes montagnes d'A sie en douze régions que nous considérons homogènes $d$ 'un point de vue climatique. A insi, nous pensons réduire certains biais et analyser l'influence de la couverture détritique sur une population plus homogène de glaciers, au moins du point de vue de la climatologie.

\section{Les rôles de la pente et de l'altitude moyenne des glaciers}

De façon surprenante, le bilan de masse ne dépend que très peu de la couverture de débris des glaciers. En revanche, la pente du bas du glacier et son altitude moyenne sont de bien meilleurs indicateurs du bilan de masse dans la plupart des régions des hautes montagnes d'A sie. On observe que les glaciers qui ont les bilans de masse les plus négatifs sont ceux qui

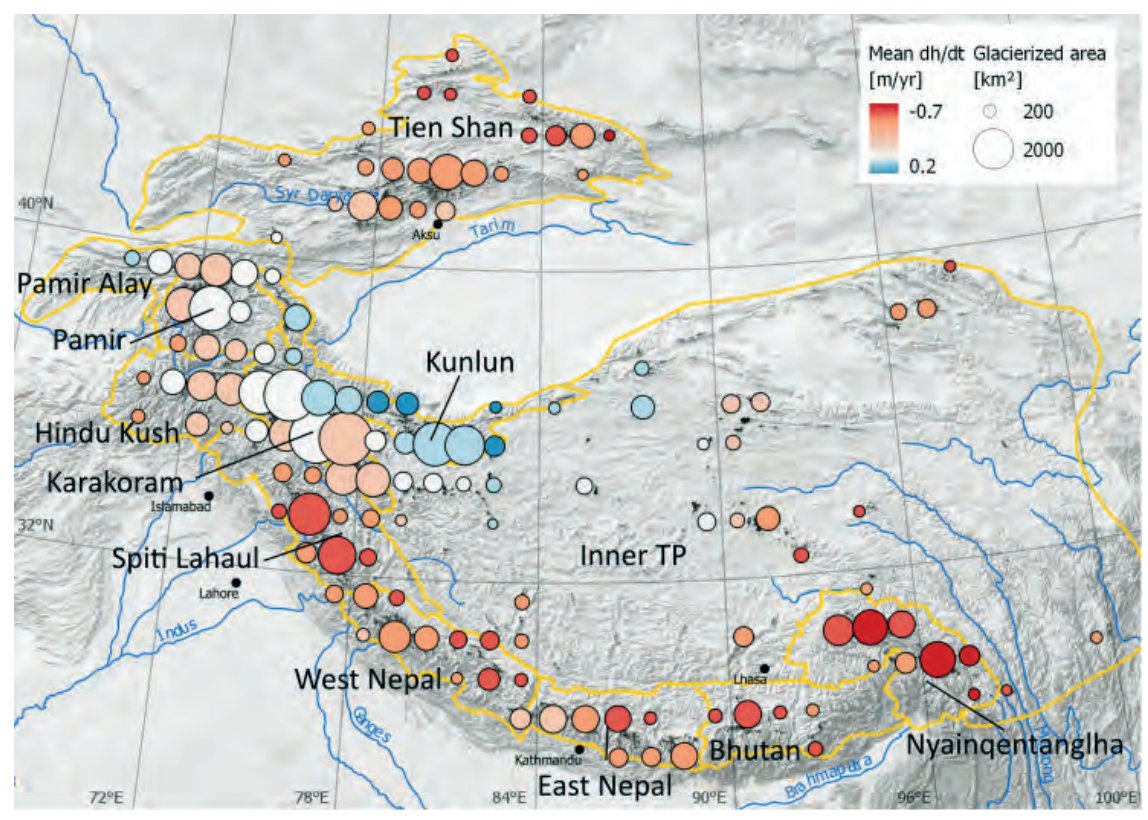

Figure 8. Carte des changements d'épaisseur des glaciers des hautes montagnes d'Asie. Chaque point représente le changement d'épaisseur moyen des surfaces englacées pour une zone de $1^{\circ}$ par $1^{\circ}$. 
ont une pente faible sur leur partie basse et qui sont situés à basse altitude (B run et al., 2019). L es rares études qui se sont penchées sur la question ont été conduites dans les A l pes et ont trouvé des résultats similaires sur l'influence de ces deux facteurs.

L'interprétation n'est pas complètement claire, mais les relations morphologie glaciaire et bilan de masse sont en général interprétées en termes de sensibilité au changement de température/précipitation ou en termes de temps de réponse. L es glaciers situés à basse altitude sont plus sensibles aux augmentations de température. En effet, ils subissent une fonte saisonnière plus importante, qui doit être compensée par des précipitations plus importantes en altitude. De plus, les glaciers à faible pente ont un temps de réponse plus long que les glaciers à forte pente, et dans un climat qui se réchauffe on s'attend à ce qu'ils soient plus loin de leur état d'équilibre et donc qu'ils aient des bilans plus négatifs.

\section{Glaciers couverts vs glaciers non couverts}

Une autre façon d'explorer ces résultats est de classifier les glaciers selon deux catégories: couverts et non couverts de débris. Dans la littérature, il n'y a pas consensus sur la définition même de ces glaciers noirs. Nous avons donc fait appel à des glaciologues experts pour classer 100 glaciers dans l'une ou l'autre de ces catégories. Nous avons ainsi déterminé que le seuil optimal de séparation entre ces deux catégories correspondait à une couverture de débris de $19 \%$ de la surface. Dans sept des douze régions étudiées, les glaciers couverts de débris ont des bilans de masse qui ne sont pas statistiquement différents des glaciers non couverts (figure 9). Cela signifie qu'ils répondent de façon très similaire au changement climatique récent et donc qu'ils ont probablement des sensibilités au changement de température similaires sur les courtes échelles de temps (16 ans pour notre étude).

Ces résultats interpellent, car, il y a quelques années, beaucoup de glaciologues auraient intuitivement répondu qu'en raison du caractère isolant des débris l'évolution des glaciers couverts devait être relativement découplée du climat ou du moins retardée par rapport à l'évolution des glaciers non couverts. N otamment, on pensait que les bilans de masse des glaciers couverts auraient dû être plus proches de zéro que ceux des glaciers non couverts dans une région donnée, ce qui n'est pas ce que nous avons observé.

\section{Interdépendance du bilan de masse de surface, débris et dynamique}

\section{Glaciers couverts et climat}

Un des apports principaux de ces travaux a été de faire prendre conscience que les glaciers couverts de débris sont probablement des indicateurs sensibles des changements climatiques, malgré une fonte réduite sur leur langue. Des interprétations récentes proposent que les glaciers noirs et glaciers blancs répondent via des mécanismes différents à un changement climatique. Les glaciers non couverts vont répondre par un recul de leur front, alors que les glaciers noirs s'amincissent sur l'ensemble de leur langue (figure 10). Sur l'échelle de temps relativement courte que nous avons étudiée, ces mécanismes différents conduisent pourtant à des bilans de masse équival ents pour des raisons que nous ne comprenons pas à l' heure actuelle.

Pour mieux comprendre ces observations, il faudrait réussir à modéliser en détail (ou paramétrer) les processus qui contrôlent la fonte des langues couvertes. Pour mieux caractériser et modéliser ces différents processus, il est nécessaire d'instrumenter de nouveaux sites pour mieux quantifier la contribution de la fonte sous les débris, des falaises de glace ou des lacs supra-glaciaires.

\section{Évolution à long terme}

La robustesse de la modélisation de ces processus est d'autant plus importante si on cherche à modéliser et à comprendre l'évolution des glaciers noirs sur des échelles de temps de plusieurs centaines voire milliers d'années, à la fois dans le passé et dans le futur. Sur des échelles de temps plus longues, il faut également prendre en compte les rétroactions entre bilan de masse de surface, dynamique de l'écoulement glaciaire et transport des débris. Par exemple, pour les glaciers des hautes montagnes d'A sie, l'amincissement des glaciers s'accompagne d'un ralentissement de leur écoulement ( $D$ eheca et al., 2019). Les glaciers deviennent ainsi moins efficaces pour évacuer les débris qu'ils transportent et, en conséquence, la proportion de leur surface couverte de débris augmente. Le changement de l'état de surface a des conséquences sur le bilan de masse qui a, à son tour, un effet sur la dynamique du glacier. Si sur de courtes échelles de temps (16 ans) les glaciers noirs et les glaciers blancs répondent de façon similaire en termes de bilan de masse, il est peu probable que cela se vérifie sur de grandes échelles de temps. II y a donc une vraie nécessité à comprendre ces rétroactions bilans de masse, transport de débris et dynamique glaciaire.

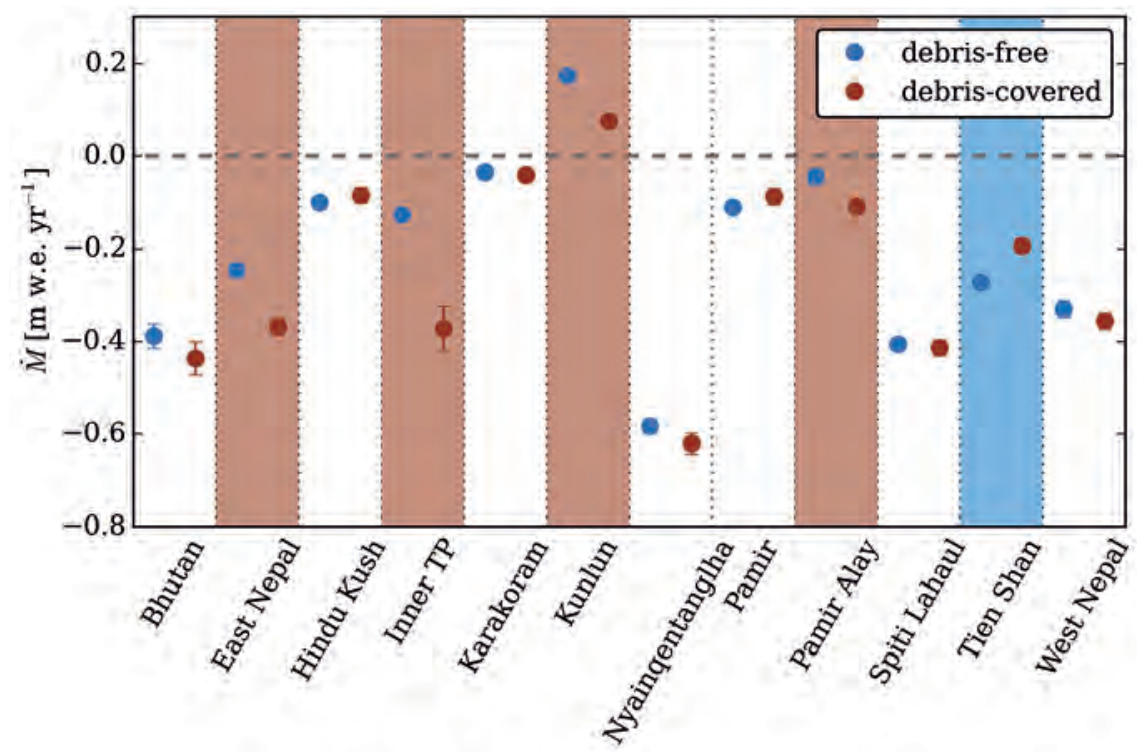

Figure 9. Moyenne des bilans de masse (en m équivalent eau/an) des glaciers noirs (points marrons) et des glaciers non couverts (points bleus) pour chaque région. Les barres d'erreurs représentent l'erreur standard et sont la plupart du temps plus petites que la taille des points. Les régions où les glaciers noirs ont un bilan statistiquement plus négatif sont colorées en marron, celles où c'est l'opposé sont colorées en bleu et les régions où la différence n'est pas significative ne sont pas colorées. 


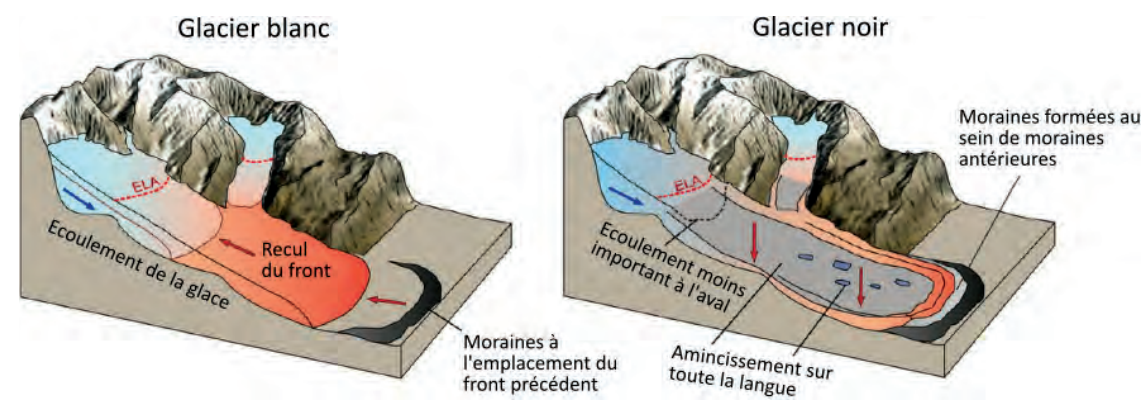

Figure 10. Réponse d'un glacier blanc et d'un glacier noir à une augmentation de l'altitude de la ligne d'équilibre (ELA), qui correspond à un réchauffement climatique. Schéma adapté de Rowan (2017).

\section{Conclusion}

Nous avons réalisé de nombreuses observations de terrain et par satellite pour chercher à mieux comprendre l'influence de la couverture de débris sur le bilan de masse des glaciers des hautes montagnes d'A sie. Les allersretours entre petite et grande échelle n'ont pas toujours été faciles à maîtriser, mais ils nous ont été nécessaires pour orienter nos questionnements, enrichir nos pistes de réflexions et nous aider à remettre en question nos hypothèses initiales.

Les glaciers noirs restent un sujet d'étude un peu à part en glaciologie. A I'heure actuelle, la relation entre glaciers noirs et climat n'est pas complètement comprise. II est donc difficile de prévoir avec certitude quelle sera l'évolution de ces glaciers dans le cadre du réchauffement climatique. M ême s'il semble peu probable que les nouvelles connaissances sur les glaciers noirs remettent complètement en cause les chiffres avancés pour l'évolution future des glaciers des hautes montagnes d'A sie, on peut s'attendre à des révisions de l'année ou de la décennie du pic des débits des rivières dans les régions où les glaciers sont fortement couverts de débris.

\section{Remerciements}

Je remercie le jury du prix André Prud'homme et l'association M étéo et Climat pour l'intérêt porté à mes travaux de thèse. Je remercie aussi toutes les personnes avec qui j'ai eu le plaisir de collaborer et de vivre de très belles aventures, que ce soit au Népal ou bien au chaud dans un bureau. Un grand merci à David Salas y M élia, à Olivier B oucher et à M ichel Zelvelder pour la relecture de cet article.

\section{Bibliographie}

Berthier E., Wagnon P., Arnaud Y., Vincent C., 2016. Les glaciers himalayens vont-ils tous disparaître avant 2035 ? La Météorologie, 95, 46-53. doi: 10.4267/2042/61617

Brun F., Berthier E., Wagnon P., Kääb A., Treichler D., 2017. A spatially resolved estimate of High Mountain Asia glacier mass balances from 2000 to 2016. Nat. Geosci., 10, 668673. doi: 10.1038/ngeo2999

Brun F., Wagnon P., Berthier E., Shea J.M., Immerzeel W.W., Kraaijenbrink P.D.A., Vincent C., Reverchon C., Shrestha D., Arnaud Y., 2018. Ice cliff contribution to the tongue-wide ablation of Changri Nup Glacier, Nepal, central Himalaya. The Cryosphere, 12, 3439-3457, doi: 10.5194/tc-12-3439-2018

Brun F., Wagnon P., Berthier E., Jomelli V., Maharjan S.B., Shrestha F., Kraaijenbrink P.D.A., 2019. Heterogeneous influence of glacier morphology on the mass balance variability in High Mountain Asia. J. Geophys. Res. Earth Surface, 124, 1331-1345. doi: 10.1029/2018JF004838

Dehecq A., Gourmelen N., Gardner A.S., Brun F., Goldberg D., Nienow P.W., Berthier E., Vincent C., Wagnon P., Trouvé E., 2019. Twenty-first century glacier slowdown driven by mass loss in High Mountain Asia. Nat. Geosci., 12, 22-27. doi: 10.1038/s41561-018-0271-9

Evatt G.W., Abrahams I.D., Heil M., Mayer C., Kingslake J., Mitchell S.L., Fowler A.C., Clark C.D., 2015. Glacial melt under a porous debris layer. J. Glaciol., 61, 825-836. doi: 10.3189/2015JoG14J235

Kraaijenbrink P.D.A., Bierkens M.F.P., Lutz A.F., Immerzeel W.W., 2017. Impact of a global temperature rise of 1.5 degrees Celsius on Asia's glaciers. Nature, 549, 257-260. doi: 10.1038/nature23878

Østrem G., 1959. Ice melting under a thin layer of moraine, and the existence of ice cores in moraine ridges. Geografiska Annaler, 41, 228-230

Rowan A.V., 2017. The "Little Ice Age" in the Himalaya: A review of glacier advance driven by Northern Hemisphere temperature change. The Holocene, 27, 292-308. doi: $10.1177 / 0959683616658530$

Sakai A., Fujita K., 2017. Contrasting glacier responses to recent climate change in high-mountain Asia. Scientific Reports, 7, 13717. doi: 10.1038/s41598-017-14256-5

Sakai A., Nakawo M., Fujita K., 1998. Melt rate of ice cliffs on the Lirung Glacier, Nepal Himalayas, 1996. Bull. Glacier Res., 16, 57-66.

Scherler D., Wulf H., Gorelick N., 2018. Global assessment of supraglacial debris-cover extents. Geophys. Res. Lett., 45, 11798-11805. doi: 10.1029/2018GL080158

Vincent C., Wagnon P., Shea J.M., Immerzeel W.W., Kraaijenbrink P., Shrestha D., Soruco A., Arnaud Y., Brun F., Berthier E., Sherpa S.F., 2016. Reduced melt on debris-covered glaciers: investigations from Changri Nup Glacier, Nepal. The Cryosphere, 10, 1845-1858. doi: 10.5194/tc-10-1845-2016 